\title{
Similarities in the Computed Tomography Appearance in a1-Antitrypsin Deficiency and Smoking-Related Chronic Obstructive Pulmonary Disease in a Smoking Collective
}

\author{
Philip Konietzke $^{\mathrm{a}-\mathrm{c}}$ Bertram Jobst ${ }^{\mathrm{a}-\mathrm{c}}$ Willi L. Wagner ${ }^{\mathrm{a}-\mathrm{c}}$ Inga Jarosch ${ }^{\mathrm{d}}$ \\ Ralph Graber ${ }^{\mathrm{e}}$ Klaus Kenn ${ }^{d}$ Hans-Ulrich Kauczor ${ }^{\mathrm{a}-c}$ Mark O. Wielpütz ${ }^{\mathrm{a}-c}$ \\ a Department of Diagnostic and Interventional Radiology, University Hospital of Heidelberg, Heidelberg, Germany; \\ ${ }^{\mathrm{b}}$ Translational Lung Research Center (TLRC) Heidelberg, Member of the German Center for Lung Research (DZL), \\ Heidelberg, Germany; ' Department of Diagnostic and Interventional Radiology with Nuclear Medicine, Thoraxklinik

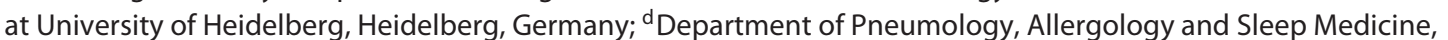

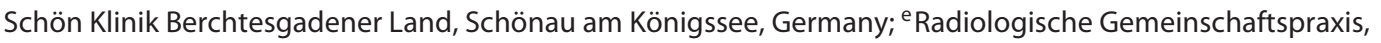 \\ Bad Reichenhall, Germany
}

\section{Keywords}

Chronic obstructive pulmonary disease $\cdot$ a1-Antitrypsin

deficiency · Imaging · Differentiation · Smoker

\begin{abstract}
Background: Emphysematous destruction of lung parenchyma visible in computed tomography (CT) can be attributed to chronic obstructive pulmonary disease (COPD) or to a1-antitrypsin deficiency (AATD). Objectives: We evaluated if visual semiquantitative phenotyping of CT data helps identifying individuals with AATD in a group of smokers with severe emphysema and airflow limitation. Method: $n=14$ patients with AATD and $n=15$ with COPD and a minimum of 10 pack years underwent $C T$, clinical assessment, and fullbody plethysmography. The extent and type of emphysema as well as large and small airway changes were rated semiquantitatively for each lobe using a standardized previously published scoring system. Lastly, a final diagnosis for each patient was proposed. Results: AATD had a significantly low-
\end{abstract}

\section{KARGER}

() 2018 S. Karger AG, Basel

E-Mail karger@karger.com

www.karger.com/res er mean emphysema score than COPD, with $8.9 \pm 3.4$ versus $11.9 \pm 3.2(p<0.001)$, respectively. Within both groups, there was significantly more emphysema in the lower lobes $(p<$ 0.05-0.001). The COPD group showed an upper- and middlelobe predominance of emphysema distribution when compared to the AATD group $(p<0.001)$. Centrilobular (CLE) and panlobular (PLE) emphysema patterns showed a uniform distribution within both groups, with a CLE predominance in the upper lung and a PLE predominance in the lower lung regions. AATD and COPD both showed significantly more airway changes in lower lobes compared to upper lobes ( $p=$ 0.05-0.001), without significant differences between both groups. Conclusion: The typical emphysema distribution patterns seen on CT traditionally assigned to AATD and COPD were of little use in discriminating both entities. Also, airway changes could not contribute to a more precise differentiation. We conclude that a concise standardized phenotyping-driven approach to chest CT in emphysema is not sufficient to identify patients with AATD in a cohort of smokers with advanced emphysema.

(c) 2018 S. Karger AG, Basel 


\section{Introduction}

a1-antitrypsin deficiency (AATD) is an autosomal recessive disorder in which homozygosis for the so-called PiZZ mutation genetic defect of Alpha-1 Antitrypsin (A1AT) causes accumulation of a1-protease in liver cells resulting in inflammation and cirrhosis [1-3] and predisposition to the early-onset development of emphysema. Chronic obstructive pulmonary disease (COPD) is a lung disease characterized by chronic obstruction of airflow that interferes with normal breathing. Cigarette smoke is the most important factor for the development of COPD and causes significant comorbidities $[4,5]$. However, the correlation between the amount of pack years and the severity of emphysema on chest computed tomography (CT) scans is weak [6]. Furthermore, there may be unique genetic differences among individuals leading to greater susceptibility to the adverse effects of cigarette smoke [7]. Genetic factors have subsequently been attributed to specific patterns and distribution of emphysema $[8,9]$. AATD and COPD share morphological features of emphysematous destruction of lung parenchyma and airway changes on CT. The Fleischner Society recently defined different patterns of emphysematous parenchymal destruction, which may be distinguished by CT [10]. Centrilobular emphysema (CLE) is the most common type of smokingrelated emphysema and it is usually upper lobe dominant [11], whereas panlobular emphysema (PLE) specially refers to diffuse emphysematous destruction and is strongly associated with low circulating levels of A1AT $[12,13]$. Paraseptal emphysema (PSE) and bullae are also associated with smoking, often with marked thickening of the walls of proximal bronchi and bronchioles, suggesting a significant airway inflammatory component [10]. Importantly, in smokers with AATD, mixtures of panlobular and centrilobular patterns may coexist [14].

Although bronchiectasis is associated with recurrent infections in AATD [15], the overall role of airway changes in AATD patients remains unclear. COPD is characterized by airflow obstruction caused by emphysema or airway narrowing, or both [16]. Small airways disease contributes to the clinical manifestations of COPD and progressively increases with GOLD classifications $[17,18]$. However, it is not fully understood which CT parameters best reflect the severity of airways disease [19]. Cigarette smoke, which is the major risk factor for COPD, induces inflammation in the lung parenchyma and the peripheral airways [20]. Visual phenotyping, as commonly used in clinical routine, refers to a standardized semiquantitative assessment of the extent of emphysema and airway changes [10]. Imaging- driven phenotyping may help to more accurately guide specific therapy than solely relying on pulmonary function testing (PFT) [21]. It is assumed that there is an emphasis of emphysema on the upper lobes in smoking-related COPD, whereas AATD mainly involves the lower lobes. In addition, the dominant emphysema pattern in COPD is usually CLE, while PLE is typical for AATD [10]. It is the aim of the present study to evaluate whether visual semiquantitative phenotyping of CT data may help to identify individuals with AATD in a group of smokers with severe emphysema and airflow limitations.

\section{Materials and Methods}

\section{Study Design}

$n=14$ patients with AATD and $n=15$ with COPD and a minimum of 10 pack years underwent CT, clinical assessment, and full-body plethysmography. Extent and pattern of emphysema and large and small airway changes were rated semiquantitatively for each lobe using a standardized previously published scoring system [22]. Furthermore, the predominant emphysema patterns were reported. Lastly, a final diagnosis was proposed based on commonly used distinctive features like upper lobe CLE in smoking-related COPD and PLE in the lower lobes in AATD.

\section{Patient Cohort}

The population for the present study was retrospectively selected from a cohort of $n=101$ patients with AATD homozygous for PiZZ and $n=101$ COPD patients homozygous for PIMM, who were part of a larger prospective clinical trial (Clinicaltrials.gov NCT01380626). Every subject gave written informed consent to examination and data processing. The study was approved by the Bavarian ethics committee (No. 09010). The aim was to form two groups as equal as possible in terms of age, gender distribution, smoking history, and lung function parameters (Table 1). Included were patients with a stable COPD (forced expiratory volume in $1 \mathrm{~s}$ [FEV1] $<50 \%$ predicted and a BMI between 20 and 30) if they already were characterized by the PiMM or PiZZ genotype. The main inclusion criterion for the present population was the availability of a clinically indicated CT scan (i.e., not part of the prospective trial) within 1 year of clinical assessment and a smoking history of at least 10 pack years. Exclusion criteria were disease exacerbation within the last 30 days, acute coronary syndrome, use of anticoagulant medication, and any disability that prevented attendance at follow-up examinations. Fourteen patients with AATD and 15 with COPD met the criteria.

\section{Computed Tomography and Standardized Image Analysis}

A thin-slice lung kernel dataset was available for semiquantitative assessment in all patients, either as conventional incremental high-resolution CT or as overlapping thin-slice reconstructions of helical CT data. Slice thickness varied between 1.0 and $1.5 \mathrm{~mm}$. Images were reviewed for diagnostic quality on a dedicated workstation. Emphysema and airway changes were visually assessed independently by two radiologists, one with $>20$ years of experience in chest imaging and airway disease, the second reader with $>4$ years of experience in chest imaging. Both readers were blinded for 
Table 1. Patient data and pulmonary function testing

\begin{tabular}{lcc}
\hline & AATD & COPD \\
\hline$n$ & 14 & 15 \\
Male/female & $9 / 5$ & $10 / 5$ \\
Age, years & $53.1 \pm 7.3$ & $59.7 \pm 7.2$ \\
Pack years & $37.4 \pm 16.8$ & $41.2 \pm 23$ \\
FEV1, L/s & $1.13 \pm 0.45$ & $0.92 \pm 0.26$ \\
FEV1, \% & $32.09 \pm 8.27$ & $30.34 \pm 8.09$ \\
TLC, \% & $149.92 \pm 16.25$ & $131.37 \pm 17.02$ \\
RV/TLC, \% & $190.96 \pm 23.03$ & $181.54 \pm 23.68$ \\
DLCO, \% & $40.11 \pm 15.48$ & $38.52 \pm 13.05$ \\
$\mathrm{PaO}_{2}, \mathrm{~mm} \mathrm{Hg}$ & $57.82 \pm 4.88$ & $58.8 \pm 8.03$ \\
\hline
\end{tabular}

Patient age and average pack years are given for the AATD and the COPD collective.

the clinical diagnosis using a standardized, modified scoring sheet as published by the COPDGene CT Workshop Group [22] (see supplementary material there). Essentially, emphysema severity was rated semiquantitatively on a five-point scale for each lobe, e.g., right upper lobe (RUL), right middle lobe (RML), right lower lobe (RLL), left upper lobe (LUL), left lingula (LLi), and left lower lobe (LLL), as follows: $<5 \%=0,5-25 \%=1,26-50 \%=2,51-75 \%$ $=3,>75 \%=4$. The predominant pattern, centrilobular or panlobular, was scored for each individual lobe. PSE and bullae were reported for right and left lung, if present, but did not add to the emphysema score, since the focus was on PLA and CLE as typical emphysema patterns for AATD and COPD. Abnormalities of the trachea and the left and right main-stem bronchi were rated on a 2 -point scale, with no abnormality $=0$ and abnormality present $=$ 1. Large intrapulmonary airways, e.g., segmental and subsegmental bronchi, were evaluated for bronchiectasis and wall thickening with a similar 2-point scale in a lobe-based approach. Small airways disease with centrilobular nodules as well as mosaic attenuation were rated as not present $=0$ and present $=1$, also in a lobebased approach. Hence, the overall emphysema score could range from 0 to a maximum score of 24, the overall airway score from 0 to 27. Finally, the reader decided based on the overall visual assessment whether the patient shows airway or emphysema dominant disease, and whether he suspects AATD or not.

\section{Pulmonary Function Testing}

Patients underwent full-body plethysmography for measurements of total lung capacity (TLC), residual volume to TLC ratio (RV/TLC), and FEV1. Further, diffusion capacity for carbon monoxide (DLCO) and arterial oxygen partial pressure $\left(\mathrm{PaO}_{2}\right.$ in $\mathrm{mm}$ $\mathrm{Hg}$ ) were measured. Lung function test results for TLC, RV/TLC, FEV1/\%, and DLCO are expressed as a percentage of predicted values. The lung function values were used to exclude structural differences between both groups.

\section{Analysis}

All data were recorded in a dedicated database (Excel ${ }^{\circledR}$, Microsoft Corp., Redmond, WA, USA), and statistical analysis was conducted using SigmaPlot ${ }^{\circledR}$ software (Systat Software GmbH, Erkrath, Germany). Data is displayed as mean \pm standard deviation.
Table 2. Inter-reader agreement

\begin{tabular}{|c|c|c|c|}
\hline & Reader 1 & Reader 2 & $\kappa$ \\
\hline \multicolumn{4}{|l|}{ Emphysema } \\
\hline \multicolumn{4}{|l|}{ AATD } \\
\hline Total lung & 124 & 124 & 0.870 \\
\hline RUL & 22 & 22 & 0.877 \\
\hline RML & 16 & 16 & 0.756 \\
\hline RLL & 27 & 27 & 0.856 \\
\hline LUL & 19 & 19 & 0.825 \\
\hline LLi & 14 & 15 & 0.868 \\
\hline LLL & 26 & 25 & 0.872 \\
\hline \multicolumn{4}{|l|}{ COPD } \\
\hline Total lung & 183 & 173 & 0.846 \\
\hline RUL & 28 & 28 & 0.854 \\
\hline RML & 30 & 29 & 0.843 \\
\hline RLL & 35 & 33 & 0.874 \\
\hline LUL & 27 & 25 & 0.845 \\
\hline $\mathrm{LLi}$ & 29 & 26 & 0.793 \\
\hline LLL & 34 & 32 & 0.815 \\
\hline \multicolumn{4}{|l|}{ Airways } \\
\hline \multicolumn{4}{|l|}{ AATD } \\
\hline Total airways & 118 & 118 & 0.809 \\
\hline Large airways & 77 & 79 & 0.835 \\
\hline Small airways & 41 & 39 & 0.805 \\
\hline \multicolumn{4}{|l|}{ COPD } \\
\hline Total airways & 106 & 114 & 0.832 \\
\hline Large airways & 66 & 68 & 0.836 \\
\hline Small airways & 77 & 79 & 0.843 \\
\hline
\end{tabular}

Inter-reader agreement for emphysema and airways scores in the AATD and COPD group. Simple weighted kappa coefficients were used to assess inter-reader agreement.

Nonparametric tests were employed for further analysis, i.e., Wilcoxon rank-sum test. Simple weighted kappa coefficients were used to assess inter-reader agreement. Kappa was interpreted as an indication that agreement was slight when $0-0.2$, fair when greater than 0.2 to 0.4 , moderate when greater than 0.4 to 0.6 , substantial when greater than 0.6 to 0.8 , and near perfect when greater than 0.8 to 1.0 , in accordance with Landis and Koch [23]. For subsequent analysis, the mean of the scores assigned by the two readers was calculated to achieve a consensus. The Spearman rank order correlation coefficient $r_{s}$ was calculated for semiquantitative CT data versus PFT. A $p$ value $<0.05$ was considered statistically significant.

\section{Results}

\section{Inter-Reader Agreement}

As shown in Table 2, inter-reader agreement for emphysema scores was comparable in both groups with $\kappa=$ 0.870 for AATD and $\kappa=0.846$ for COPD. Acceptable in- 
Fig. 1. Emphysema severity distribution in AATD and COPD. Emphysema severity was rated semiquantitatively for each lobe on a 5-point scale for AATD and COPD represented by the scale on the right. All patients except 1 AATD patient showed emphysema. COPD patients showed higher emphysema scores in general.

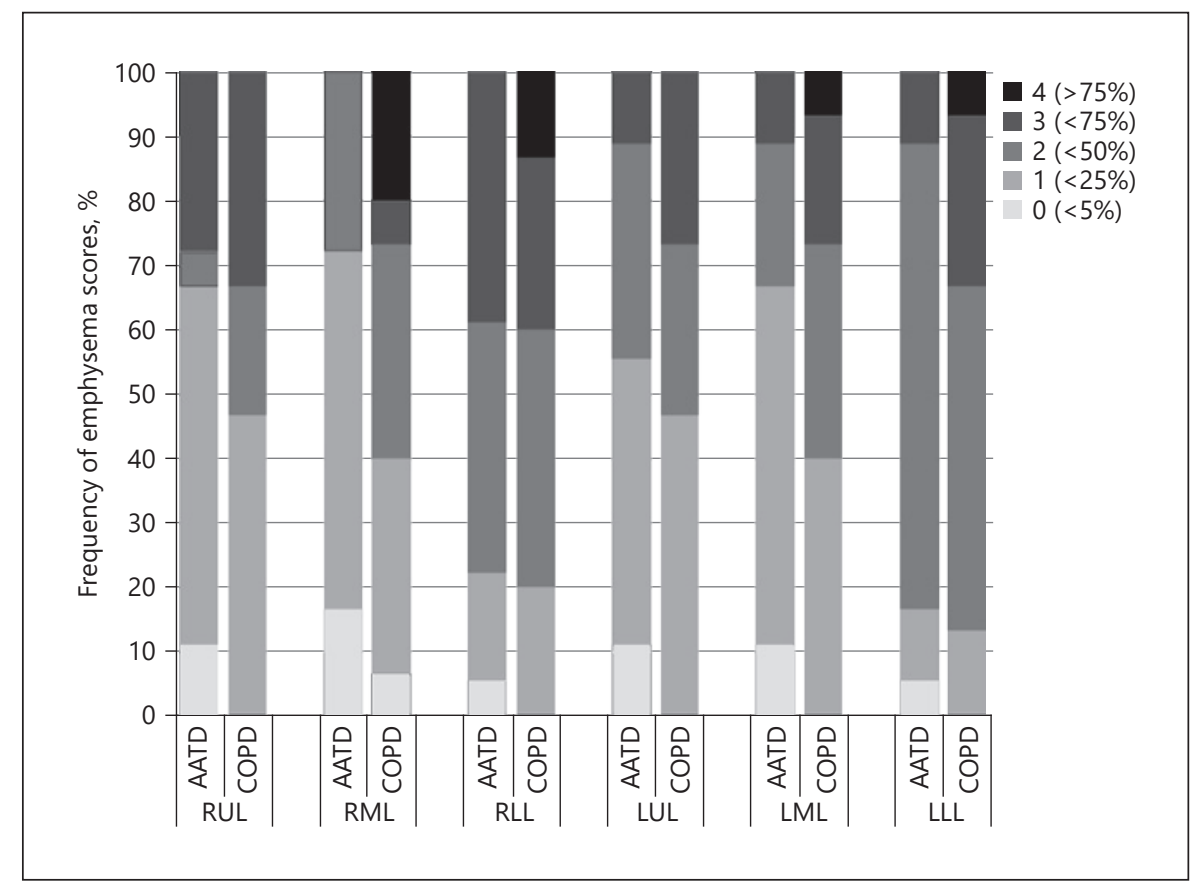

ter-reader agreement was found for airway changes with $\kappa=0.809$ for AATD and $\kappa=0.832$ for COPD.

\section{Emphysema Distribution Patterns in AATD and COPD}

All AATD and COPD patients showed emphysema with different degrees of severity. The mean emphysema score for the total lung was significantly higher in COPD than in AATD (Table 3). Furthermore, COPD patients showed overall more severe emphysema, and $20 \%$ of all COPD patients reached the highest emphysema score of $4(>75 \%)$ in the RML, whereas none of the AATD patients reached maximum emphysema scores (Fig. 1). Regarding the lobe-based analysis, there were no significant differences between the individual lobes in AATD and COPD, except for the RML and the lingula, which had a significantly higher emphysema score in COPD. If the individual lung lobes are combined to upper lung regions (e.g., RUL, LUL, RML, and LLi) and lower lung regions (e.g., RLL and LLL), a significant higher emphysema score was found for the upper lung regions in COPD compared to AATD (Table 3). Looking at the distribution within the AATD and COPD group, there was a significantly higher emphysema score in the lower lung regions compared to the upper lung regions in both groups.

Overall, CLE and PLE patterns were equally common. Interestingly, both groups showed a CLE pattern more
Table 3. Emphysema scores in AATD and COPD

\begin{tabular}{lrrl}
\hline & AATD & COPD & \multicolumn{1}{l}{$p$} \\
\hline Total lung & $8.9 \pm 3.4$ & $11.9 \pm 3.2$ & 0.001 \\
RUL & $1.6 \pm 1.1$ & $1.9 \pm 0.8$ & 0.423 \\
RML & $1.1 \pm 0.5$ & $2.0 \pm 1.0$ & 0.036 \\
RLL & $1.9 \pm 0.9$ & $2.3 \pm 0.9$ & 0.501 \\
LUL & $1.4 \pm 0.7$ & $1.7 \pm 0.8$ & 0.348 \\
LLi & $1.1 \pm 0.5$ & $1.8 \pm 0.9$ & 0.015 \\
LLL & $1.8 \pm 0.6$ & $2.2 \pm 0.7$ & 0.146 \\
Lung regions & & & \\
$\quad$ Upper lung regions & $1.3 \pm 0.8$ & $1.9 \pm 0.9$ & 0.001 \\
$\quad$ Lower lung regions & $1.9 \pm 0.8$ & $2.2 \pm 0.8$ & 0.168 \\
\hline$p \quad$ & 0.001 & 0.043 & \\
\hline
\end{tabular}

Means of emphysema score and standard deviations for the total lung and upper (e.g., RUL, LUL, RML, and LLi) and lower lung regions (e.g., RLL and LLL) as well as the individual lobes in AATD and COPD.

frequently in the upper, and PLE more frequently in the lower lung regions. The distribution of emphysema patterns in the upper lung regions did not differ considerably between both groups. Within the COPD group, there was a notably higher frequency of CLE pattern in the lower lung regions compared to AATD (Fig. 2, 3). 


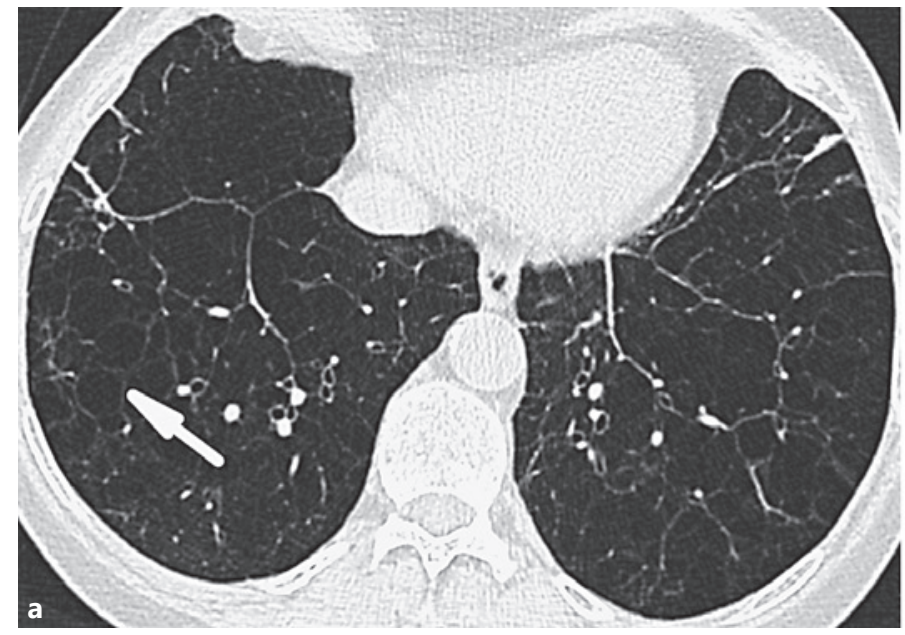

Fig. 2. Comparison of panlobular emphysema AATD and confluent emphysema in COPD. Axial CT image (a) shows a representative example of PLE related to AATD. The lower lungs show widespread confluent areas of hyperlucency overspanning one or several lobules. Some lobules, outlined by intact interlobular septa,

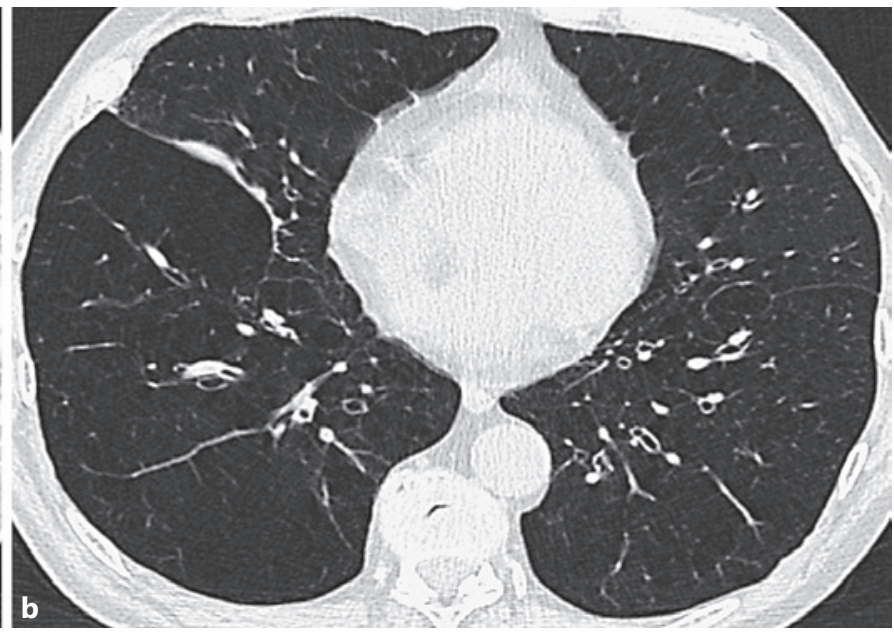

appear hyperinflated. There is also distortion of pulmonary architecture. On the axial CT image on the right side (b), confluent CLE in COPD is shown. Multiple lucencies that span several lobules can be identified, but interlobular septa are not visible. PLE and confluent CLE are thus hard to differentiate.

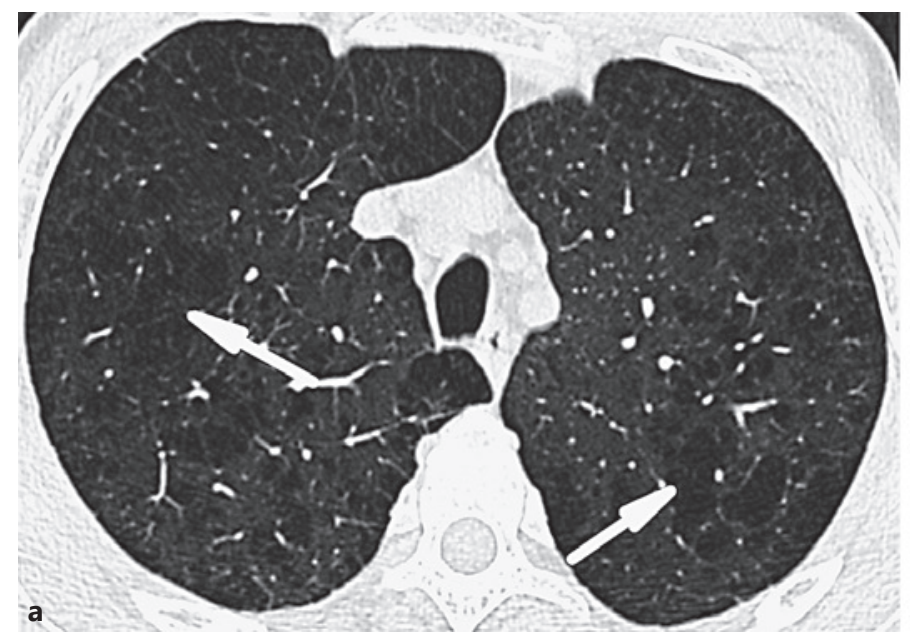

Fig. 3. Centrilobular emphysema in AATD and COPD. Moderate CLE in patients with AATD 4 (a) and a patient with COPD (b). Both CT scans show multiple well-defined centrilobular lucencies

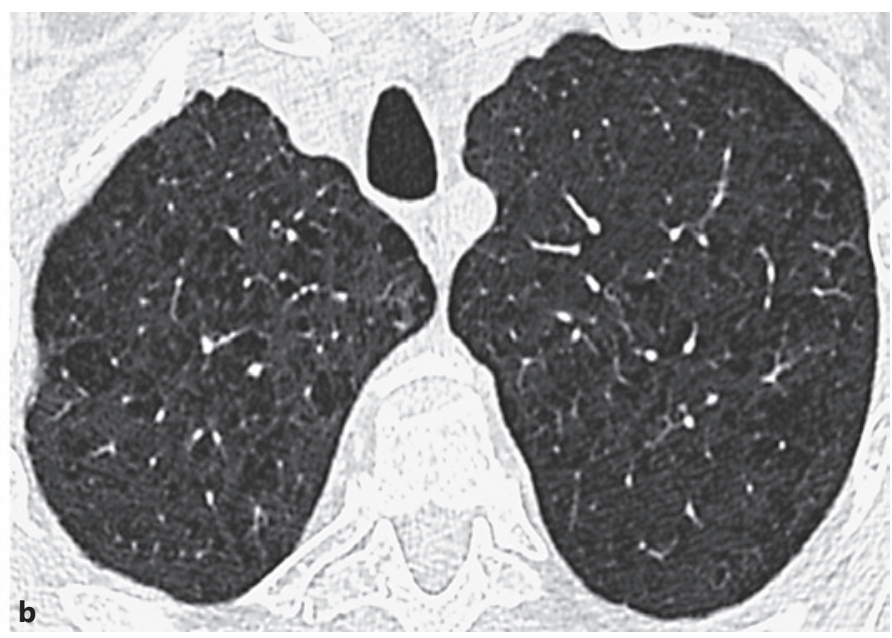

that occupy more than $5 \%$ of the upper lung zone. In AATD, some areas of confluent hyperlucencies are visible, which may be early PLE or confluent CLE.
PSE was detected in $79 \%$ of AATD and $40 \%$ of COPD patients and exclusively found bilaterally in the upper lobes. Bullae were also restricted to the upper lobes and were present in $29 \%$ of AATD patients and in $40 \%$ of COPD patients. The differences for PLE and the prevalence of bullae were not significant $(p=0.107 ; p=0.173$ ) (Table 4).

Similarities in the CT Appearance in AATD and COPD in Smokers

\section{Airway Changes Do Not Discriminate AATD from} COPD Patients

Airway changes were visible in all AATD and COPD patients. Tracheal abnormalities were reported in 57\% of all AATD and in 53\% of all COPD patients mostly associated with abnormalities in both main bronchi. The overall airway changes showed no notable differ-

Respiration 2018;96:231-239 
Table 4. Prevalence of emphysema patterns

\begin{tabular}{|c|c|c|c|c|}
\hline & \multicolumn{2}{|l|}{ AATD } & \multicolumn{2}{|l|}{ COPD } \\
\hline & CLE & PLE & CLE & PLE \\
\hline Lobes with emphysema & $38(49.35 \%)$ & $38(50.65 \%)$ & $43(51.69 \%)$ & $46(48.31 \%)$ \\
\hline \multicolumn{5}{|l|}{ Lung regions } \\
\hline Upper lung region & 8.375 & 6 & 8 & 6.75 \\
\hline Lower lung region & 2.75 & 10.75 & 5.5 & 9.5 \\
\hline \multicolumn{5}{|l|}{ Lobes } \\
\hline RUL & 9 & 3.5 & 8.5 & 6.5 \\
\hline RML & 8 & 4.5 & 8.5 & 5.5 \\
\hline RLL & 3.5 & 10 & 5.5 & 9.5 \\
\hline LUL & 8.5 & 4 & 6 & 9 \\
\hline $\mathrm{LLi}$ & 8 & 4.5 & 9 & 6 \\
\hline LLL & 2 & 11.5 & 5.5 & 9.5 \\
\hline
\end{tabular}

Prevalence of CLE and PLE patterns for all lobes and all individual lung lobes in AATD and COPD. The first row shows the total prevalence for CLE and PLE. Single lobes and lobes without emphysema are not listed.

Table 5. Severity of airway changes in AATD and COPD

\begin{tabular}{|c|c|c|c|c|c|c|c|c|c|}
\hline & \multicolumn{3}{|c|}{ Total airways } & \multicolumn{3}{|c|}{ Large airways } & \multicolumn{3}{|c|}{ Small airways } \\
\hline & AATD & COPD & $p$ & AATD & COPD & $p$ & AATD & COPD & $p$ \\
\hline RUL & $1.5 \pm 1.1$ & $1.1 \pm 1.1$ & 0.351 & $0.9 \pm 0.7$ & $0.5 \pm 0.7$ & 0.145 & $0.6 \pm 0.7$ & $0.6 \pm 0.6$ & 0.851 \\
\hline RML & $1.4 \pm 0.9$ & $0.9 \pm 0.8$ & 0.174 & $1.0 \pm 0.6$ & $0.7 \pm 0.7$ & 0.280 & $0.4 \pm 0.5$ & $0.2 \pm 0.4$ & 0.556 \\
\hline RLL & $1.8 \pm 0.8$ & $1.7 \pm 1.1$ & 0.928 & $1.1 \pm 0.5$ & $1.1 \pm 0.7$ & 0.982 & $0.5 \pm 0.7$ & $0.6 \pm 0.6$ & 0.868 \\
\hline LLL & $1.7 \pm 0.9$ & $1.8 \pm 1.2$ & 0.637 & $1.3 \pm 0.6$ & $1.1 \pm 0.8$ & 0.594 & $0.5 \pm 0.5$ & $0.7 \pm 0.7$ & 0.310 \\
\hline \multicolumn{10}{|l|}{ Lung regions } \\
\hline Upper lung regions & $1.3 \pm 1.0$ & $0.9 \pm 0.9$ & 0.083 & $0.8 \pm 0.7$ & $0.6 \pm 0.7$ & 0.059 & $0.4 \pm 0.6$ & $0.4 \pm 0.5$ & 0.885 \\
\hline Lower lung regions & $1.7 \pm 0.9$ & $1.8 \pm 1.1$ & 0.817 & $1.2 \pm 0.6$ & $1.1 \pm 0.7$ & 0.722 & $0.5 \pm 0.5$ & $0.7 \pm 0.6$ & 0.538 \\
\hline$p$ & 0.027 & 0.001 & & 0.009 & 0.001 & & 0.401 & 0.044 & \\
\hline
\end{tabular}

Prevalence of airway abnormalities and severity scores for AATD and COPD for the total lung, RUL, RML, RLL, LUL, LLi, and LLL are shown. Airway changes in large airways and in small airways as well as the total lung were considered separately. In addition, the statistical differences between the upper and lower lung regions are given, for AATD versus COPD (vertical) and within the AATD and COPD group (horizontal).

ences between the AATD and the COPD group. Looking at the distribution within the AATD and COPD group, there were significantly higher airway scores in the lower lung regions compared to the upper lung regions for large airway changes and small airway changes (Table 5).

\section{Final Diagnosis Based on Visual Phenotyping}

The final diagnosis stated by the readers based on only the imaging findings was correct for $73 \%$ of all patients for the first reader and for $69 \%$ of all patients for the second reader $(\kappa=0.734)$. In the AATD, both readers were correct in 93 and $100 \%$ of the cases $(\kappa=0.965)$, whereas in COPD, only 53 and $40 \%$ of the patients were diagnosed correctly $(\kappa=0.737)$. AATD was overdiagnosed in this study using visual phenotyping alone. In both groups, AATD $(\kappa=0.737)$ and COPD $(\kappa=0.762)$, the diagnosis "airway dominant disease" was given to 3 patients and in 11 , respectively 12 patients, the diagnosis "emphysema dominant disease" was given. 
Table 6. Correlation of emphysema and airways scores with PFT

\begin{tabular}{lrrrrrr}
\hline & FEV1 & FEV1\% & TLC & RV/TLC & DLCO & $\mathrm{PaO}_{2}$ \\
\hline $\begin{array}{l}\text { Emphysema } \\
\text { AATD }\end{array}$ & & & & & & 0.500 \\
COPD & -0.340 & -0.291 & -0.449 & -0.036 & 0.468 & 0.018 \\
Airways & & 0.287 & 0.235 & -0.064 & 0.102 & 0.400 \\
AATD & 0.387 & 0.168 & -0.077 & -0.088 & 0.648 & -0.073 \\
COPD & 0.600 & 0.014 & -0.401 & -0.116 & -0.029 & \\
\hline
\end{tabular}

The Spearman rank order correlation coefficient $\left(r_{s}\right)$ was calculated for semiquantitative CT data versus PFT.

\section{Pulmonary Function Testing and Correlation with} Scores

All patients demonstrated severe abnormalities in PFT indicating advanced obstructive airways disease. There was no significant difference between both groups regarding the PFT parameters with the exception of TLC being significantly higher in the AATD group (Table 1). Emphysema score and PFT parameters did not show significant correlations for AATD or COPD (Table 6). Regarding the visual airway score, there was a significant correlation with FEV1 in COPD patients (Table 6).

\section{Discussion}

It has been proposed that the predominant pattern of emphysema may help in distinguishing AATD and smoking-related COPD [10]. PLE is the typical pattern in AATD and it refers to a diffuse emphysematous destruction across the lung lobule $[13,24]$. PLE is thought to affect all acini within a lung lobule equally, and the overall extent and severity of alveolar destruction is believed to be milder than in CLE. Smoking-associated CLE is morphologically characterized by small areas of low attenuation in the center of a lung lobule, surrounded by normal lung parenchyma, usually with a small blood vessel in the center of the lesion [25]. This CT pattern correlates well with pathological findings of centrilobular emphysematous destruction $[26,27]$. CLE may progress in a "confluent" disease pattern and finally in "advanced destructive emphysema" (ADE) [10]. Surprisingly, in our study, PLE had also a lower lobe predominance in COPD. This seemingly conflicting result may have several reasons. PLE is largely indistinguishable from "confluent" CLE and ADE on CT, as demonstrated by the fact that the term PLE has been previously used to describe this form of advanced
CLE in COPD patients $[14,28]$. Furthermore, mild PLE is difficult to identify on CT [10], and lastly, in cigarette smokers, mixtures of PLE and CLE can be found within the same lungs [14]. In summary, two major factors complicate the discrimination of AATD from COPD based on emphysema patterns: Firstly, "confluent" CLE can simulate a PLE pattern in COPD patients and secondly, CLE can be found in smokers with AATD. PSE is characterized by subpleural and peribronchovascular foci of low attenuation separated by thickened interlobular septa and frequently associated with mild signs of fibrosis [29]. In our study population, PSE was only present in the upper lobes without significant difference between AATD and COPD. Bullae are found in all subtypes of emphysema but are most commonly associated with PSE [30]. They are often located in the upper lobes in both PSE and CLE but are more evenly distributed in patients with ADE [31]. Bullae were present in 20\% of all AATD deficiency patients and in $53 \%$ of all COPD. Bullae may therefore be indicative of COPD.

The relative lobar distribution of emphysema may also assist in discriminating AATD and COPD. In our study, AATD and COPD both showed the highest emphysema scores in the lower lobes, which is typical for AATD deficiency, in which emphysema, if occurring as advanced PLE, usually has a lower lobe predominant distribution $[31,32]$. In our small study population, we did not detect upper lung predominance for emphysema in COPD as it may be expected [10]. Nevertheless, COPD showed a significantly higher emphysema score for combined upper lung regions (RUL, LUL, LLi, RML vs. lower lobes) ( $p=$ 0.001 ) when compared to AATD. This may be helpful in distinguishing COPD form AATD. Genetic determinates may be a possible explanation for the untypical high emphysema scores in the basal lung regions in the COPD group, but at the present time, this cannot be proven [9]. 
Furthermore, COPD had significantly higher overall emphysema score and showed more severe types of emphysema.

Airway disease is commonly found in all forms of emphysema, but also occurs in the absence of emphysema as predominant phenotype of COPD [10]. In our study, the overall airway changes tend to be higher in AATD than in COPD, showing no significant difference, however. There was lower lobe predominance of airway disease in both groups. Large airway disease is associated with abnormalities of the trachea and central bronchi, bronchial wall thickening, and bronchiectasis. Especially bronchial wall thickening is commonly observed in heavy cigarette smokers [33], due to bronchial inflammation and remodeling. It may be visually identified on CT by a relative increase in bronchial wall thickness compared with the bronchial lumen and with the diameter of adjacent pulmonary arteries [34]. Bronchiectasis is defined as a dilated bronchial lumen relative to the adjacent pulmonary artery [30]. The reported CT-detected prevalence of bronchiectasis in COPD patients ranges from 27 to $58 \%$ $[11,35]$. In AATD, a potential association between AATD and the presence of both bronchiectasis and bronchial wall thickening $[31,36]$ has been suggested. Small airway disease is often an important major component of both emphysema-predominant disease and airway-predominant disease involving larger airways. Isolated small airway disease can also occur as primary expression of COPD [10]. In our study, large airway changes were found more often in AATD than in COPD, but the difference was not significant. The prevalence of small airway changes in AATD and COPD was even. Overall, large and small airway changes could not help distinguishing between COPD and AATD.
The limitations of our current study include the small numbers of patients in both groups. In addition, it should be noted that consistent scan protocols would have been desirable to obtain the highest level of comparability between the individual studies. A further possible drawback is the one-time evaluation of the data, whereby a possible intrareader deviation was neglected. However, we wanted to address the general problem of distinguishing both entities in a smoking collective and we assume that a second read would not have provided any additional information.

As a conclusion, our data demonstrates that a concise standardized phenotyping-driven approach to chest CT in emphysema is not sufficient to identify patients with AATD in a cigarette-smoking collective with advanced COPD. The reason for this is that the classic distinguishing features like lower lobe predominant PLE in AATD and upper lobe predominant CLE in COPD are superimposed by smoking-induced changes. Although there are useful findings like the presence of bullae and upper lobe emphysema predominance in COPD, especially heavy smokers might have extensive upper lobe CLE in AATD and ADE simulating "true" PLE in COPD. Therefore, radiologists are likely to overcall the presence of AATD when reporting patients with advanced emphysema because of the high similarity of confluent CLE and "true" PLE.

\section{Disclosure Statement}

The authors have nothing to disclose.

\section{References}

1 Perlmutter DH, Brodsky JL, Balistreri WF, Trapnell BC: Molecular pathogenesis of alpha-1-antitrypsin deficiency-associated liver disease: a meeting review. Hepatology 2007; 45:1313-1323.

2 Wood AM, Stockley RA: Alpha one antitrypsin deficiency: from gene to treatment. Respiration 2007;74:481-492.

3 Baraldo S, Balestro E, Bazzan E, Tine ME, Biondini D, Turato G, et al: Alpha-1 antitrypsin deficiency today: new insights in the immunological pathways. Respiration 2016;91: 380-385.
4 Golpe R, Martin-Robles I, Sanjuan-Lopez P, Cano-Jimenez E, Castro-Anon O, MengualMacenlle N, et al: Prevalence of major comorbidities in chronic obstructive pulmonary disease caused by biomass smoke or tobacco. Respiration 2017;94:38-44.

5 Mannino DM, Buist AS: Global burden of COPD: risk factors, prevalence, and future trends. Lancet 2007;370:765-773.

6 Bellomi M, Rampinelli C, Veronesi G, Harari S, Lanfranchi F, Raimondi S, et al: Evolution of emphysema in relation to smoking. Eur Radiol 2010;20:286-292.
7 Lokke A, Lange P, Scharling H, Fabricius P, Vestbo J: Developing COPD: a 25 year follow up study of the general population. Thorax 2006;61:935-939.

8 DeMeo DL, Hersh CP, Hoffman EA, Litonjua AA, Lazarus R, Sparrow D, et al: Genetic determinants of emphysema distribution in the national emphysema treatment trial. Am J Respir Crit Care Med 2007;176:42-48.

9 Boueiz A, Lutz SM, Cho MH, Hersh CP, Bowler RP, Washko GR, et al: Genome-wide association study of the genetic determinants of emphysema distribution. Am J Respir Crit Care Med 2017;195:757-771. 
10 Lynch DA, Austin JH, Hogg JC, Grenier PA, Kauczor HU, Bankier AA, et al: CT-definable subtypes of chronic obstructive pulmonary disease: a statement of the Fleischner Society. Radiology 2015;277:192-205.

11 Bafadhel M, Umar I, Gupta S, Raj JV, Vara DD, Entwisle JJ, et al: The role of CT scanning in multidimensional phenotyping of COPD. Chest 2011;140:634-642.

12 Laurell C, Eriksson S: The electrophoretic alpha-globulin pattern of serum in alpha 1-antitrypsin deficiency. Acta Med Scand 1963; 175:197-205.

13 Wyatt JP, Fischer VW, Sweet HC: Panlobular emphysema: anatomy and pathodynamics. Dis Chest 1962;41:239-259.

14 Kim WD, Eidelman DH, Izquierdo JL, Ghezzo H, Saetta MP, Cosio MG: Centrilobular and panlobular emphysema in smokers. Two distinct morphologic and functional entities. Am Rev Respir Dis 1991;144:1385-1390.

15 Kauczor HU, Hoffmann A, Mehdikhani H, Schunk K, Schlegel J, Mildenberger P: Bronchiectasis and infection incidence in alpha 1-antitrypsin deficiency. The value of highresolution computed tomography (in German). Rofo 1995;163:378-382.

16 American Thoracic Society: Standards for the diagnosis and care of patients with chronic obstructive pulmonary disease. Am J Respir Crit Care Med 1995;152:S77-S121.

17 Crisafulli E, Pisi R, Aiello M, Vigna M, Tzani $\mathrm{P}$, Torres A, et al: Prevalence of small-airway dysfunction among COPD patients with different GOLD stages and its role in the impact of disease. Respiration 2017;93:32-41.

18 Contoli M, Scichilone N: The contribution of small-airway abnormalities in chronic obstructive pulmonary disease clinical manifestations: more than a functional issue. Respiration 2017;93:81-82.

19 Nambu A, Zach J, Schroeder J, Jin G, Kim SS, Kim YI, et al: Quantitative computed tomography measurements to evaluate airway dis- ease in chronic obstructive pulmonary disease: relationship to physiological measurements, clinical index and visual assessment of airway disease. Eur J Radiol 2016;85:21442151.

20 Deveci F, Murat A, Turgut T, Altuntas E, Muz $\mathrm{MH}$ : Airway wall thickness in patients with COPD and healthy current smokers and healthy non-smokers: assessment with high resolution computed tomographic scanning. Respiration 2004;71:602-610.

21 Lim HJ, Weinheimer O, Wielputz MO, Dinkel J, Hielscher T, Gompelmann D, et al: Fully automated pulmonary lobar segmentation: influence of different prototype software programs onto quantitative evaluation of chronic obstructive lung disease. PLoS One 2016; 11:e0151498.

22 COPDGene CT Workshop Group, Barr RG, Berkowitz EA, Bigazzi F, Bode F, Bon J, et al: A combined pulmonary-radiology workshop for visual evaluation of COPD: study design, chest $\mathrm{CT}$ findings and concordance with quantitative evaluation. COPD 2012;9:151-159.

23 Landis JR, Koch GG: The measurement of observer agreement for categorical data. Biometrics 1977;33:159-174.

24 McDonough JE, Yuan R, Suzuki M, Seyednejad N, Elliott WM, Sanchez PG, et al: Smallairway obstruction and emphysema in chronic obstructive pulmonary disease. New Engl J Med 2011;365:1567-1575.

25 Murata K, Itoh H, Todo G, Kanaoka M, Noma $\mathrm{S}$, Itoh T, et al: Centrilobular lesions of the lung: demonstration by high-resolution CT and pathologic correlation. Radiology 1986; 161:641-645.

26 Foster WL, Jr., Gimenez EI, Roubidoux MA, Sherrier RH, Shannon RH, Roggli VL, et al: The emphysemas: radiologic-pathologic correlations. Radiographics 1993;13:311-328.

27 Hruban RH, Meziane MA, Zerhouni EA, Khouri NF, Fishman EK, Wheeler PS, et al: High resolution computed tomography of in- flation-fixed lungs. Pathologic-radiologic correlation of centrilobular emphysema. Am Rev Respir Dis 1987;136:935-940.

28 Smith BM, Austin JH, Newell JD, Jr., D'Souza BM, Rozenshtein A, Hoffman EA, et al: Pulmonary emphysema subtypes on computed tomography: the MESA COPD study. Am J Med 2014;127:94 e7-e23.

29 Satoh K, Kobayashi T, Misao T, Hitani Y, Yamamoto Y, Nishiyama Y, et al: CT assessment of subtypes of pulmonary emphysema in smokers. Chest 2001;120:725-729.

30 Hansell DM, Bankier AA, MacMahon $\mathrm{H}$, McLoud TC, Muller NL, Remy J: Fleischner Society: glossary of terms for thoracic imaging. Radiology 2008;246:697-722.

31 Guest PJ, Hansell DM: High resolution computed tomography (HRCT) in emphysema associated with alpha-1-antitrypsin deficiency. Clin Radiol 1992;45:260-266.

32 Copley SJ, Wells AU, Müller NL, Rubens MB, Hollings NP, Cleverley JR, et al: Thin-section $\mathrm{CT}$ in obstructive pulmonary disease: discriminatory value. Radiology 2002;223:812819

33 Park JS, Brown KK, Tuder RM, Hale VA, King Jr TE, Lynch DA: Respiratory bronchiolitisassociated interstitial lung disease: radiologic features with clinical and pathologic correlation. J Comput Assist Tomogr 2002;26:13-20.

34 Ooi GC, Khong PL, Chan-Yeung M, Ho JC, Chan PK, Lee JC, et al: High-resolution CT quantification of bronchiectasis: clinical and functional correlation. Radiology 2002;225: 663-672.

35 Martinez-Garcia MA, Soler-Cataluna JJ, Donat Sanz Y, Catalan Serra P, Agramunt Lerma $\mathrm{M}$, Ballestin Vicente J, et al: Factors associated with bronchiectasis in patients with COPD. Chest 2011;140:1130-1137.

36 Cuvelier A, Muir JF, Hellot MF, Benhamou D, Martin JP, Benichou J, et al: Distribution of alpha(1)-antitrypsin alleles in patients with bronchiectasis. Chest 2000;117:415-419. 